\title{
DESLOCAMENTO DE ÓLEO POR ÁGUA EM UM MICROCANAL COM CAVIDADE DE QUINAS ARREDONDADAS
}

\author{
S. F. RIGATTO, O. J. ROMERO*
$\begin{gathered}\text { Programa de Pós-Graduação em Energia, Universidade Federal do Espírito Santo - UFES, Campus São Mateus } \\ \text { oldrich.romero@ufes.br }\end{gathered}$ \\ Submetido 14/08/2017 - Aceito 25/12/2018 \\ DOI: $10.15628 /$ holos.2018.5234
}

\section{RESUMO}

No presente estudo é analisado numericamente, com auxílio do software Ansys Fluent, a mobilização de óleo mediante injeção de água em uma geometria bidimensional formado por placas paralelas com uma microcavidade quadrada que, de forma simplificada, representa as gargantas e poros da rocha-reservatório portadora de óleo. O objetivo fundamental é avaliar a influência da curvatura da quina no processo de mobilização do óleo. Para isso, o efeito de três raios de curvatura são comparados com a quina formada por um ponto. O problema é transiente, bifásico, com forças capilares dominantes sobre as viscosas e as de inercia. Os resultados obtidos, para o caso com quina aguda, é coincidente com o trabalho de Coelho, Pena e Romero
\end{abstract}

(2016). A análise dos casos considerados permitem destacar que ao final do processo de deslocamento uma fração de óleo continuará trapeado na microcavidade. Também, para a geometria considerada e independente do raio de curvatura da quina, existem três regiões distintas ao se avaliar a fração volumétrica média de óleo. A primeira região em que a redução de óleo é acentuada. Uma segunda região onde a retirada de óleo torna-se cada vez mais lenta. E uma terceira que identifica a saturação de óleo residual. Finalmente, o processo se torna mais eficiente quanto maior for o raio de curvatura da quina do microporo. Esta relação, pelo menos para os casos analisados, é linear.

PALAVRAS-CHAVE: Microcavidade, quinas arredondadas, escoamento bifásico, VOF, eficiência de deslocamento.

\section{OIL/WATER DISPLACEMENT IN A MICROCHANNEL WITH SQUARE CAVITY FORMED BY A ROUNDED CORNER}

\begin{abstract}
In this work, the oil displacement process due to water injection in a two-dimensional geometry formed by parallel plates with a micro square cavity that, in simplified form, represents the pores of the oil reservoir rock is analyzed numerically. This was achieved with the Ansys Fluent software that uses the finite volume method. The main aim of this paper is the investigation of the influence of a rounded corner of the geometry in the oil displacement process. In the multiphasic, transient, Newtonian flow, capillary forces prevailing over the inertial and viscous forces. The results obtained, for the case with square corners, coincide with the work of Coelho, Pena e Romero (2016). The analysis shows that, for the four cases considered, at the
\end{abstract}

end of the oil displacement process a portion of the oil will remain trapped in the microcavity. Additionally, for the considered geometry, independent of the radius of curvature of the rounded corners, there are three distinct regions when evaluating the average oil volume fraction. A first region, where the reduction of oil is accentuated, then a second region, where the oil displacement becomes slower, and finally a third region, identified by the saturation of the residual oil. The process becomes more efficient as the radius of curvature of the micropore with rounded corners increases. This relation, at least for the cases analyzed, is linear.

KEYWORDS: Microcavity, rounded corners, two-phase flow, VOF, displacement efficiency. 


\section{INTRODUÇÃO}

No processo produtivo do petróleo, uma das etapas mais desafiadoras é a que envolve o escoamento no meio poroso. A rocha-reservatório, portadora do óleo que se deseja escoar para a superfície, se encontra a centenas de metros no subsolo, e seu acesso é indireto utilizando o poço perfurado e outros procedimentos. As informações que podem ser obtidas desse ambiente são sempre incertas, entretanto isso depende do tempo de vida do local. A confiabilidade dos dados coletados melhora com o tempo e é bastante representativa quando o reservatório deve ser abandonado, onde, claro, estes dados são já de utilidade quase nula.

Além dos poucos dados e da incerteza acentuada destes, principalmente quando estudos estão sendo realizados para desenvolver de forma mais eficiente o campo petrolífero, o escopo de estudo envolve um domínio que é praticamente impossível de se definir corretamente devido à tortuosidade dos caminhos pelos quais os fluidos escoam (Figura 1). Aliado a isso, tem-se diversas escalas, saindo das centenas de quilômetros para a escala microscópica de alguns micrometros. A velocidade de movimentação dos fluidos típica no reservatório é de $1 \mathrm{ft} / \mathrm{dia}$ (Green \& Willhite, 1998). Esta combinação de microescala e velocidades baixas resultam em que no balanço de forças existentes no ambiente multifásico do meio poroso envolvendo fluido imiscíveis seja dominada pelas forças de capilaridade. As forças viscosas mostram sua importância quando se trata de óleos pesados.

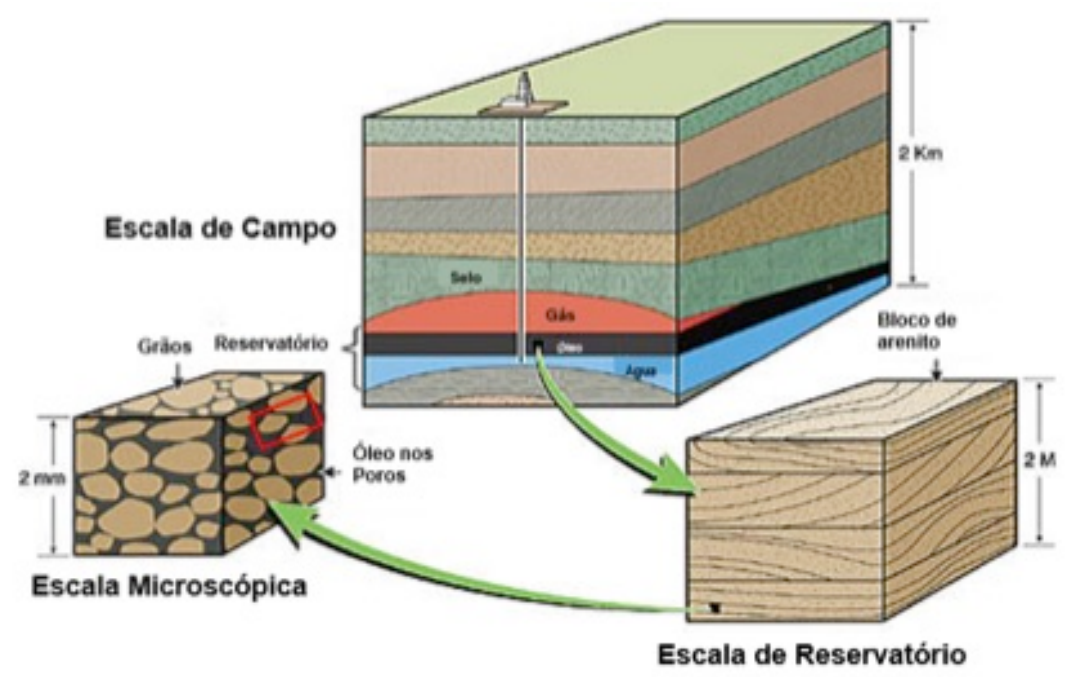

Figura 1: Estrutura porosa presentes em um reservatório de petróleo típico. Fonte: Santos, Romero, Meneguelo e Ribeiro (2016).

Forças capilares dominantes significam existência de fenômenos como snap off, que originam bolhas de fluido não molhante em uma fase continua molhante, e o efeito Jamin, que exige gradientes de pressão elevados para movimentação das bolhas formadas potencializando a retenção do óleo (Romero \& Fejoli, 2015). O trapeamento do óleo no interior da rochareservatório é um dos fatores principais para que a eficiência do processo extrativo seja baixo.

Devido à economicidade e praticidade, a forma mais difundida de melhorar o baixo fator de recuperação da produção dos mecanismos naturais é injetar água no reservatório. $A$ injeção de água para empurrar o óleo é viável desde que o óleo não seja muito viscoso. O parâmetro que se utiliza para mensurar esta característica é a razão de mobilidades $(M)$, a qual compara a 
mobilidade da água $\left(\lambda_{w}\right)$ com a mobilidade do óleo $\left(\lambda_{o}\right)$. Como a mobilidade é o inverso da viscosidade, fica claro que enquanto mais viscoso o óleo for, menos mobilidade apresentará, resultando em $\lambda_{w}>\lambda_{o}$, ou de forma equivalente, processo com razão de mobilidades desfavorável $M \gg 1$. Este comportamento é indesejado pois a água injetada encontra caminhos preferenciais (a bem conhecida instabilidade denominada digitação viscosa) perfurando o banco de óleo para ser produzida, é o breakthrough. O óleo fica para atrás e cada vez mais água é coletada em superfície. O BSW - Basic Sediments and Water começa a aumentar até valores tão altos quanto $95 \%$. Além da injeção de água, outras alternativas, enquadradas nos métodos especiais de recuperação (EOR - Enhanced Oil Recovery), podem ser utilizadas, entretanto requerem maior investimento e domínio de tecnologias diferenciadas por parte da empresa operadora do campo. Seja qual for a técnica utilizada, uma quantidade de óleo residual ficará trapeado no reservatório. De forma simples, isso deve-se a que nem todo o volume do reservatório pode ser contatado pelo fluido injetado, e mesmo da região contatada nem todo o óleo pode ser deslocado. Portanto as eficiências volumétrica, ou de varrido, $E_{V}$ e a de deslocamento, ou microscópica, $E_{D}$ são menores do que 1.

A eficiência da extração de petróleo é quantificado comparando quanto óleo foi retirado do volume que inicialmente existia no reservatório. Este parâmetro é o fator de recuperação $\left(f_{R}\right)$ que, de forma conveniente é resultado do produto entre a eficiência volumétrica e a eficiência de deslocamento (Green \& Willhite, 1998), isto é

$f_{R}=E_{V} E_{D}$.

A eficiência volumétrica é obtida considerando a escala macroscópica do reservatório e é objeto de estudo da simulação tradicional de reservatórios de petróleo. Já a eficiência de deslocamento trata da mobilização de óleo na escala microscópica e é mensurado comparando as saturações de óleo ao início $\left(S_{o_{-} \text {inicial }}\right)$ e ao final do processo $\left(S_{o_{-} \text {final }}\right)$, tal que

$E_{D}=1-\frac{S_{o_{-} \text {final }}}{S_{O_{-} \text {inicial }}}$.

O que influência o trapeamento? É possível mobilizar o óleo trapeado? Como ocorre a mobilização? Qual é a importância da geometria dos poros? Qual é o papel das propriedades dos fluidos envolvidos? Quais são os mecanismos envolvidos? As respostas a essas, e outras perguntas, são a motivação para o estudo que vem sendo desenvolvido no Programa de PósGraduação em Energia da Ufes no contexto da linha de pesquisa de simulação numérica de escoamentos bifásicos em microescalas.

A mobilização de óleos retidos em microcavidades têm sido estudada desde a perspectiva monofásica (Yin, 2006; Kamyabi, Ramazani \& Kamyabi, 2013; Pena, Romero \& Ribeiro, 2014; Kamyabi, Ramazani \& Kamyabi, 2015) e mais recentemente bifásica (Coelho, Pena \& Romero, 2016). A maioria das geometrias utilizadas são microcavidades quadradas com quinas agudas. Esse pressuposto não condiz com a realidade, uma vez que as microcavidades não possuem quinas formadas por ângulos retos (Romero, Scriven \& Carvalho, 2006).

Desta forma, o presente trabalho tem como objetivo analisar numericamente a influência da curvatura da quina no processo de deslocamento de óleo por água, e, dessa forma tornar a simulação mais condizente com a realidade. 


\section{ESPECIFICAÇÃO DO PROBLEMA ABORDADO}

O presente estudo é direcionado aos fenômenos identificados principalmente na escala microscópica, por tanto a eficiência macroscópica $\left(E_{V}\right)$ da Equação (1) é admitido ser unitária. A eficiência de deslocamento pode ser entendida como o desafio para movimentar o óleo retido nos poros da rocha. Entretanto, como o problema real possui geometria complexa (Figura 1), simplificações são incorporadas para viabilizar seu estudo resultando em um modelo físico simples, contudo mantendo sua essência e representatividade.

Os fluidos presentes no meio poroso apresentam propriedades e características que podem assumir valores diferentes de um ponto a outro no reservatório. $O$ óleo é formado por diversas cadeias de hidrocarbonetos, com frações de água na forma de emulsões água em óleo, sedimentos. A água injetada, que normalmente é a que é produzida junto com o óleo, apresenta sais, certo teor de óleo, gás, sedimentos e contaminantes. Adicionalmente, as propriedades dependem da pressão e temperatura. Estudar um problema bifásico, tridimensional e transiente, incorporando as características relatadas tornaria a abordagem complexa. Visando obter a solução do problema em uma escala de tempo aceitável, a geometria é simplificada e os dois fluidos são considerados livres de quaisquer mistura e com propriedades constantes.

Assim, e como esquematizado na Figura 2, a geometria é concebida como formada por duas placas planas paralelas, que representam um capilar plano de comprimento $10 h$ e altura $h$, com uma microcavidade quadrada com lados $h$, com $h$ constante é igual a 0,001 m. Toda a geometria é inicialmente preenchida com óleo de viscosidade $\mu_{o}=10^{-3} \mathrm{~kg} /(\mathrm{m} \mathrm{s})$ e massa específica $\rho_{o}=900 \mathrm{~kg} / \mathrm{m}^{3}$, na qual é injetada água de viscosidade $\mu_{w}=10^{-3} \mathrm{~kg} /(\mathrm{m} \mathrm{s})$ e massa específica $\rho_{w}=1.000 \mathrm{~kg} / \mathrm{m}^{3}$ na face esquerda do domínio (McCain Jr., 1990). As viscosidades são consideradas iguais para facilitar o entendimento dos resultados. Sendo o óleo mais viscoso, como normalmente ocorre, sua retirada da cavidade seria afetada pela razão de viscosidades, ou de forma mais ampla, pela razão de mobilidades (Green \& Willhite, 1998). Este novo cenário será objeto de futuros trabalhos que utilizarão os resultados do presente artigo como parâmetro de comparação. Como os fluidos são imiscíveis, e de acordo com a valor adotado em Coelho et al. (2016), a tensão interfacial água/óleo $\sigma_{w o}$ é considerada sendo igual a $\sigma_{w o}=7,4 \times 10^{-6} \mathrm{~N} / \mathrm{m}$. Sabe-se entretanto que a tensão interfacial é da ordem de 10 a $30 \mathrm{mN} / \mathrm{m}$ (Green \& Willhite, 1998; McCain Jr., 1990). O valor utilizado neste trabalho se justifica porque o parâmetro adimensional número de capilaridade deve ser menor do que 1, implicando um escoamento com forças de capilaridade dominantes conforme valor na Tabela 2, subseção §3.6. A presença da cavidade quadrada e a presença das forças de capilaridade e forças viscosas, tornam o deslocamento do óleo por água um escoamento complexo.

Como um meio poroso real não é formado por quinas agudas neste trabalho é incorporado o efeito do arredondamento da quina no processo de deslocamento. Três raios de curvatura, $5 \mathrm{~mm}, 10 \mathrm{~mm}$ e $25 \mathrm{~mm}$, são utilizados para a quina mais próxima do plano de entrada. Ainda na Figura 2 é possível identificar as letras entre parêntesis que representam as condições de contorno, as quais são detalhadas na subseção §3.1. 


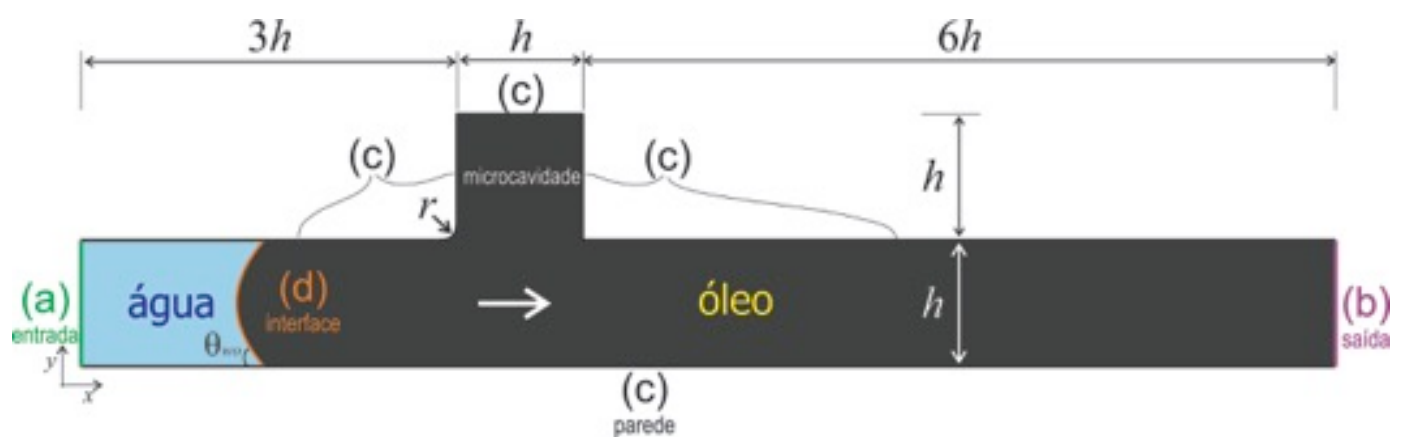

Figura 2: Representação do problema bidimensional de deslocamento de óleo por água em um microcanal com $h=$ $1 \mathrm{~mm}$, em um tempo $t$ qualquer em que a interface água/óleo se encontra perto do plano de entrada. As letras entre parêntesis representam as condições de contorno detalhadas na subseção §3.1.

Na configuração bidimensional da Figura 2 o volume de água injetada, $\mathbb{V}_{w}(t)$, e que é função do tempo, invade o domínio de volume $\mathbb{V}_{\text {total }}=(10 h \times h+h \times h) \times b=11 h^{2} b$ deslocando o fluido contido nessa região, por consequência o volume de óleo $\mathbb{V}_{o}(t)$ diminui. $O$ parâmetro $b$ é a profundidade perpendicular ao plano da figura mostrada. Uma forma de acompanhar estas variações ao longo do tempo $t$ é mediante o conceito de fração volumétrica média $\bar{\alpha}_{f}(t)$ do fluido $f$, que para a água $(f=w)$ é $\bar{\alpha}_{w}(t)=\mathbb{V}_{w}(t) / \mathbb{V}_{\text {total }}$, e para o óleo $(f=o)$ $\bar{\alpha}_{o}(t)=\mathbb{V}_{o}(t) / \mathbb{V}_{\text {total }}$. No escoamento bifásico água/óleo em estudo, a soma das frações volumétricas médias em todo instante de tempo é unitária, isto é: $\bar{\alpha}_{o}(t)+\bar{\alpha}_{w}(t)=1$.

A informação da fração de óleo, que depende do tempo é particularmente importante ao início e ao final do processo. No tempo inicial $(t=0)$ o volume de óleo preenche todo o domínio, portanto $\mathbb{V}_{o}(t=0)=\mathbb{V}_{o_{\text {_inicial }}}=\mathbb{V}_{\text {total }}$. Desta forma, para a configuração proposta, a fração volumétrica média de óleo no início do processo de deslocamento assume seu valor máximo, sendo igual a $\bar{\alpha}_{o_{-} \text {inicial }}=1$. Como a quantidade de óleo diminui, seu volume ao final da injeção $\left(t=t_{\text {final }}\right)$ é $\mathbb{V}_{o_{\text {f }} \text { inal }}$, que representa o volume de óleo retido na microcavidade. Desta forma a fração volumétrica média final de óleo é

$\bar{\alpha}_{o_{-} \text {final }}=\frac{\mathbb{V}_{o_{-} \text {final }}}{\mathbb{V}_{\text {total }}}$

este parâmetro representa a quantidade de óleo, em termos fracionários, que fica retido na cavidade.

Como o processo depende do tempo de injeção de água, é possível estabelecer o intervalo de variação da fração volumétrica média de óleo, para a configuração e condições deste estudo, como sendo

$\bar{\alpha}_{o_{-} \text {final }} \leq \bar{\alpha}_{o}(t) \leq 1$

Por outro lado, das equações apresentadas e para eficiência macroscópica unitária, temse $f_{R}=1-\bar{\alpha}_{o_{f} \text { final }}$. Nesta equação pode ser verificado que $\bar{\alpha}_{o_{-} \text {final }}$ deve ter o menor valor possível porque isso traduz quão eficiente o processo de remoção de óleo é. Como poderá ser observado no decorrer do desenvolvimento deste trabalho, $\bar{\alpha}_{o_{-} \text {final }}$ depende, em maior ou 
menor grau, de diversos parâmetros adimensionais como número de capilaridade $(\mathrm{Ca})$, razão de viscosidades $\left(\mu_{R}\right)$, ângulo de adesão $\left(\theta_{w o}\right)$, número de Reynolds $(R e)$, depende também da forma e do volume da cavidade, isto é, da geometria. Desta forma tem-se $\bar{\alpha}_{o_{-} \text {final }}=f\left(C a, \mu_{R}, \theta_{w o}, R e\right.$, geometria $)$.

\section{METODOLOGIA DE SOLUÇÃO DO PROBLEMA LÍQUIDO/LÍQUIDO}

A presença da interface, cuja forma e posição, em cada ponto do domínio e para diferentes instantes de tempo, dependem das condições operacionais, propriedades do líquido e da geometria, tornam o processo de deslocamento de óleo por água um problema complexo que requer a aplicação de técnicas numéricas específicas para sua solução. De forma geral, os métodos para abordar este tipo de problemas podem ser classificadas como de malha móvel ou de malha fixa. Os primeiros requerem a solução de equações para determinar a posição dos pontos nodais. Na segunda categoria a interface é determinada de forma indireta, resolvendo também uma equação escalar, entretanto a malha é mantida fixa, é o caso da técnica Volume Of Fluid - VOF de Hirt e Nichols (1981) utilizada neste trabalho. Em ambas abordagens equações adicionais são necessárias aumentando o tamanho da matriz a ser resolvia. Cada método apresenta suas vantagens e desvantagens, maiores detalhes das técnicas está fora do escopo deste trabalho, entretanto exemplo de aplicações são encontrados em Santos et al. (2016), Coelho et al. (2016) e Romero e Fejoli (2015).

A técnica VOF aplicada ao escoamento bidimensional, isotérmico, transiente, de dois líquidos Newtonianos, incompressíveis e imiscíveis, resolve em cada ponto do domínio as equações da continuidade, Equação (5), da quantidade de movimento linear, Equação (6), juntamente com as equações para determinar as frações volumétricas de cada fluido, Equações (7) e (8), respectivamente.

$\nabla \cdot \mathbf{v}=0$,

$\frac{\partial\left(\rho_{w o} \mathbf{v}\right)}{\partial t}+\nabla \cdot\left(\rho_{w o} \mathbf{v v}\right)=-\nabla p+\nabla \cdot\left(2 \mu_{w o} \mathbf{D}\right)+\rho_{w o} \mathbf{g}+\mathbf{f}_{\mathrm{vol}}$,

$\mathbf{v}$ é o vetor velocidade, $p$ é a pressão, $\mathbf{g}$ representa o vetor aceleração da gravidade com $\mathbf{D}$ o tensor taxa de deformação definido como $\mathbf{D}=\frac{1}{2}\left[\nabla \mathbf{v}+(\nabla \mathbf{v})^{T}\right]$, onde $\nabla \mathbf{v}$ é o tensor gradiente de velocidade e o sobrescrito ()$^{T}$ representa a operação transposta.

A fração volumétrica da fase óleo $\alpha_{o}$, secundária, é determinada mediante

$\frac{\partial\left(\alpha_{o} \rho_{o}\right)}{\partial t}+\nabla \cdot\left(\alpha_{o} \rho_{o} \mathbf{v}\right)=0$,

como tem-se somente dois fluidos, a fração volumétrica da água $\alpha_{w}$, que é a fase primária, é obtida diretamente de

$\alpha_{w}=1-\alpha_{o}$.

Desta forma, em cada ponto do domínio, ou nos elementos da malha quanto se apresenta em termos discretos, as frações volumétricas da água $\alpha_{w}$ e do óleo $\alpha_{o}$ podem assumir os seguintes valores:

- $\alpha_{w}=0$, significando que não existe água somente óleo, com isso $\alpha_{o}=1$; 
- $0<\left(\alpha_{w}\right.$ ou $\left.\alpha_{o}\right)<1$, quando a interface água/óleo está contida neste ponto. Neste caso um algoritmo específico é utilizado para determinar a posição e forma correta da interface no interior de cada célula;

- $\alpha_{w}=1$, quando a célula está completamente preenchida pela água, portanto sem óleo, isto é, $\alpha_{o}=0$.

Com as frações volumétricas determinadas para diversas posições e instantes de tempo, e com as massas específicas da água $\rho_{w}$ e do óleo $\rho_{o}$ sendo dados de entrada, é definida a massa específica $\rho_{w o}$ que abrange toda a região onde ocorre o escoamento dos dois fluidos, mediante

$\rho_{w o}=\alpha_{w} \rho_{w}+\alpha_{o} \rho_{o}$

esta expressão é utilizada para determinar o termo convectivo na Equação (6). Com similar argumentação, a viscosidade $\mu_{w o}$ aplicada em todo o domínio é definida como sendo

$\mu_{w o}=\alpha_{w} \mu_{w}+\alpha_{o} \mu_{o}$,

que por sua vez especifica o termo viscoso.

Adicionalmente, na Equação (6) o termo de força volumétrica $\mathbf{f}_{\mathrm{vol}}$ representa os efeitos da tensão interfacial $\sigma_{w o}$ no escoamento, e que neste trabalho é definido pelo modelo Continuum Surface Force - CSF de Brackbill, Kothe \& Zemach (1992)

$\mathbf{f}_{\mathrm{vol}}=\sigma_{w o} \kappa \frac{\rho_{w o} \nabla \alpha_{o}}{\frac{1}{2}\left(\rho_{w}+\rho_{o}\right)}$,

a curvatura $\kappa$ da interface água / óleo é obtida de $\kappa=\nabla \cdot\left(\frac{\nabla \alpha_{o}}{\left|\nabla \alpha_{o}\right|}\right)$.

\subsection{Condições de fronteira e iniciais}

No problema bidimensional em coordenadas cartesianas apresentado, tem-se cinco incógnitas $\left[u, v, p, \alpha_{w}, \alpha_{o}\right]$ e cinco equações [(5), (6-x), (6-y), (7), (8)]. Para proceder com sua solução condições de contorno devem ser especificadas. Estas condições são identificadas pelas letras (a) até (d) na Figura 2, e representam:

(a) na entrada, a velocidade da fluido injetado, que é água, é prescrito como $V_{w}=0,001$ $\mathrm{m} / \mathrm{s}$ e é mantido constante em todo o trabalho;

(b) no plano de saída a pressão é nula;

(c) parede fixa, na qual as condições de não escorregamento e de impenetrabilidade são válidas;

(d) na interface a Equação (11) é aplicada.

De acordo com as condições de fronteira consideradas, a pressão no plano de entrada é livre para aumentar ou diminuir, de tal forma e manter a vazão constante ao longo do processo de remoção do óleo.

Como condição inicial, antes de iniciar o processo de deslocamento, é considerado que o óleo preenche todo o volume em condições estáticas. 


\subsection{Algoritmos de discretização das equações}

O conjunto de equações é resolvida numericamente com o auxílio o software Ansys Fluent que utiliza o método VOF de Hirt e Nichols (1981). A escolha adequada de métodos de discretização compõe um passo importante do processo de simulação e neste estudo são baseados nos trabalhos de Santos et al. (2016) e Coelho et al. (2016). Para o acoplamento pressão-velocidade é selecionado o PISO - Pressure-Implicit with Splitting of Operators.

A avaliação dos gradientes das propriedades conservadas é obtida aplicando o método Least Squares Cell-Based. O esquema de interpolação da pressão selecionado é o PRESTO! PREssure STaggering Option. Para a quantidade de movimento o esquema utilizado é o QUICK Quadratic Upwind Implicit Differencing Convective Kinematics. E no caso da fração volumétrica o esquema utilizado é o CICSAM - Compressive Interface Capturing Scheme for Arbitrary Meshes.

\subsection{Nível de refinamento da malha}

Sete níveis de refinamento (malha $1=1.105$ elementos, malha $2=1.820$ elementos, malha $3=3.118$ elementos, malha $4=6.885$ elementos, malha $5=9.149$ elementos, malha $6=$ 12.112 elementos 5 malha $7=15.141$ elementos), com elementos não estruturados são testados. O parâmetro de comparação é a fração volumétrica média de óleo utilizando os dados do caso 4 e os resultados obtidos são apresentados na Figura 3. A diferença nas repostas ocorre a partir de $t_{D}=\sim 0,85$ que identifica o fim do trecho linear e o início do trecho não-linear. Embora a partir de $t_{D}=\sim 2$ todas as malhas colapsam em um mesmo patamar, o aumento do nível de refinamento origina uma retirada mais lenta do óleo. Contudo, a partir da malha 4 de 6.885 elementos as respostas são muito similares, sugerindo que a independência da malha foi alcançado.

Assim, o nível de refinamento selecionado para todos os casos analisados neste trabalho é a que corresponde à malha 5, de 9.149 elementos (Figura 4) com qualidade ortogonal de $87,88 \%$ e razão de aspecto de 2,08. O tempo final para analisar a fração de óleo retida deve ser longo o suficiente para não influenciar nos resultados do processo de deslocamento, neste caso $t_{D}>2$.

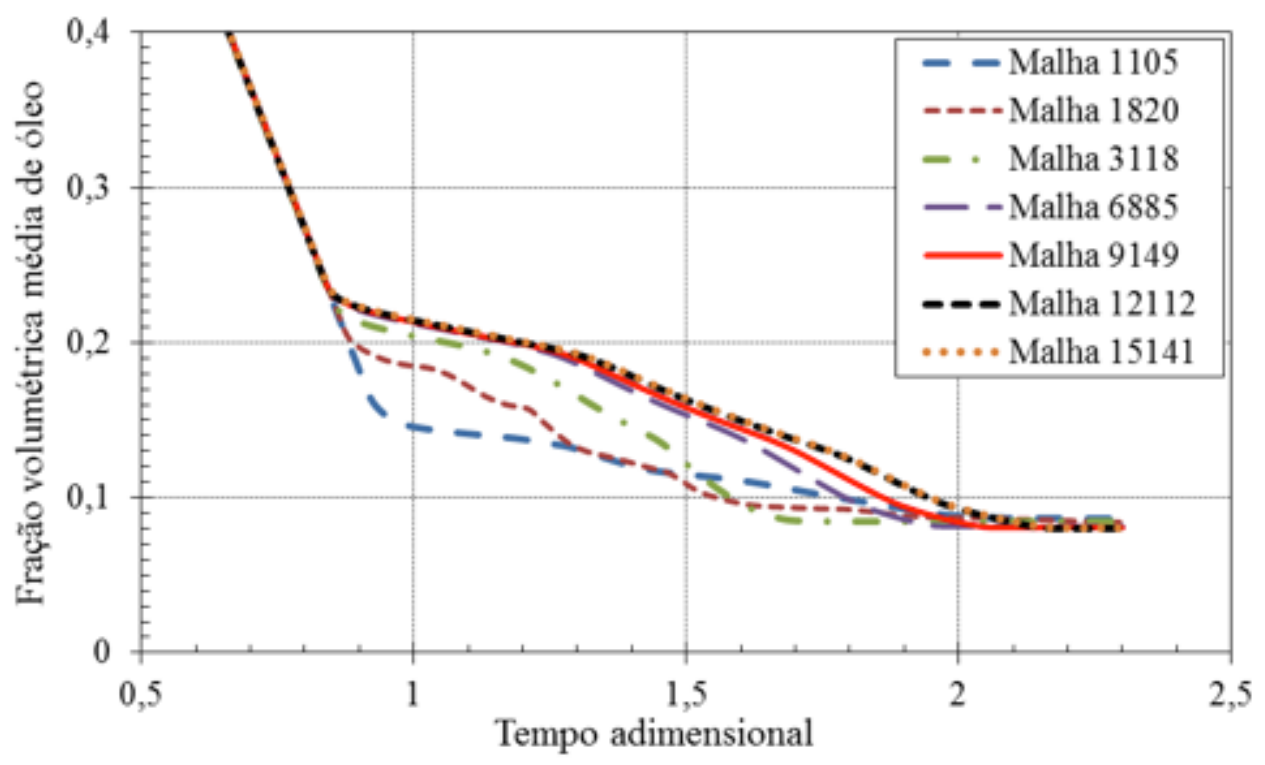

Figura 3: Teste de malha para as condições do caso 4. 


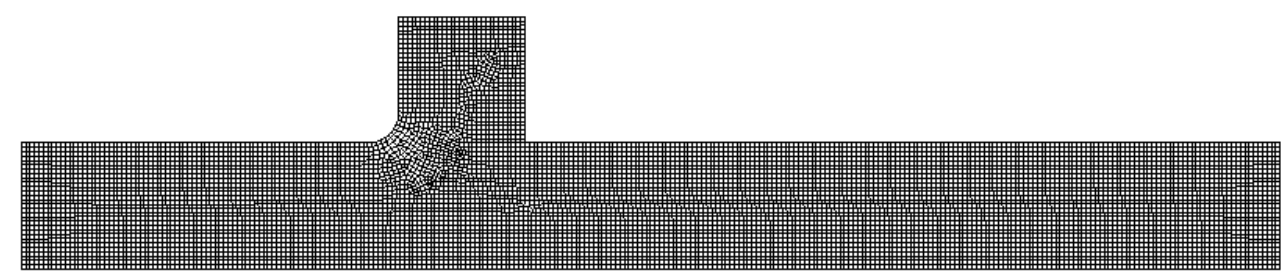

Figura 4: Malha não estruturada utilizada neste trabalho com 9.149 elementos.

\subsection{Escolha do passo de tempo}

A escolha do passo de tempo $\Delta t$ é realizada conforme recomendação de Bohacek (2010) e discutidas em Coelho et al. (2016) e Santos et al. (2016), em que três condições devem ser verificadas. Para os dados deste problema, assumindo um número de Courant $C o=0,25$ e considerando para a velocidade do elemento da malha o valor da velocidade de entrada, os valores obtidos para o passo de tempo de Courant é $\Delta t_{C F L}=0,025 \mathrm{~s}$, o de Brackbill et al. (1992) $\Delta t_{B}=0,0046 \mathrm{~s}$ e o de Galusinski e Vigneaux (2008) é $\Delta t_{V}=0,0135 \mathrm{~s}$. A condição de Brackbill exige o menor passo de tempo para garantir estabilidade no processo de solução, e é adotado neste trabalho.

\subsection{Parâmetros adimensionais}

Os parâmetros adimensionais importantes neste processo de deslocamento água/óleo podem ser agrupados em físicos, numéricos e geométricos, e são os seguintes:

- Número de capilaridade $(C a), C a=\frac{\mu_{w} V_{w}}{\sigma_{w o}}$ que se refere à importância das forças viscosas em relação às de capilaridade, $\sigma_{w o}$ representa a tensão interfacial;

- Número de Reynolds ( $R e), R e=\frac{\rho_{w} V_{w} h}{\mu_{w}}$, compara a importância das forças de inércia em relação às forças viscosas. Baixos números de Reynolds indicam escoamento laminar e valores elevados de números de Reynolds caracterizam um comportamento turbulento;

- Número de Bond (Bo), $B o=\frac{\Delta \rho g h^{2}}{\sigma_{w o}}$, também denominado número de Eötvös, caracteriza a relação das forças gravitacionais com as forças interfaciais. $\Delta \rho$ representa a diferença de massa específica entre as fases, $g$ é a aceleração da gravidade, e $h$ é a distância vertical;

- Razão de viscosidades $\mu_{R}=\frac{\mu_{o}}{\mu_{w}}$, é a razão entre a viscosidade do óleo presente no domínio e a viscosidade da água a ser injetada;

- Número de Courant (Co), Co $=V_{w} \frac{\Delta t}{\Delta x}$, é um critério que visa garantir a estabilidade numérica na resolução de problemas transientes com abordagem explícita, o qual não deve ser maior do que 1 . Onde $V_{w}$ é a velocidade média do fluido injetado, $\Delta t$ é passo de tempo e $\Delta x$ o tamanho de um elemento representativo da malha. A restrição de passo de tempo de Courant-Friedrichs-Lewy (CFL) é por tanto $\Delta t_{C F L} \approx \frac{\operatorname{Co\Delta x}}{V_{w}}$

- Tempo adimensional $\left(t_{D}\right)$, o tempo de simulação $t$, em segundos, é também adimensionalizado para $t_{D}=\frac{V_{w}, t}{\phi L}$, considerando que o fluido se desloca a uma 
velocidade média (igual à velocidade prescrita na entrada), $V_{w}$, ao longo do canal de comprimento $L=10 h$, que de forma geral tem porosidade $\phi$. Substituindo os valores conhecidos e para a porosidade unitária, tem-se $t_{D}=0,1 t$;

- Raio da quina adimensional $\left(r_{D}\right), r_{D}=\frac{r}{h}$, sendo $r$ o raio da quina, que é utilizado para introduzir mais realismo ao processo.

\subsection{Cenários considerados}

Para realizar as simulações utiliza-se 25.000 passos de tempo de 0,0046 s cada um. A média de tempo para cada simulação foi de aproximadamente 16 horas de uso contínuo em um computador Intel ${ }^{\circledR}$ Core $^{\mathrm{TM}}$ i3 - 350M CPU @ $2.27 \mathrm{GHz}$ e 3GB de RAM.

Os valores obtidos para os parâmetros adimensionais que caracterizam o fenômeno estudado são apresentados na Tabela 2.

Tabela 2: Casos estudados neste trabalho. As alterações são no raio de curvatura da quina $r_{D}$ os outros parâmetros são mantidos constantes.

\begin{tabular}{cccccccc}
\hline Caso & $r_{D}, \%$ & $\theta_{w o}$ & $\mu_{R}$ & $C a$ & $R e$ & Bo & Co \\
\hline 1 & 0 & $90^{\circ}$ & 1 & 0,135 & 1 & 132,432 & 0,131 \\
2 & 5 & $90^{\circ}$ & 1 & 0,135 & 1 & 132,432 & 0,131 \\
3 & 10 & $90^{\circ}$ & 1 & 0,135 & 1 & 132,432 & 0,131 \\
4 & 25 & $90^{\circ}$ & 1 & 0,135 & 1 & 132,432 & 0,131 \\
\hline
\end{tabular}

\section{RESULTADOS E COMENTÁRIOS}

O processo bidimensional de deslocamento de óleo por água, que é injetada no plano esquerdo a uma velocidade constante, é detalhado na Figura 5 para o caso 4 com $r_{D}=10 \%$. Conforme evidenciam as linhas de corrente ao se tornarem paralelas, o escoamento se desenvolve em um trecho curto, após percorrer uma distância menor do que $h$ no canal de entrada. Como a velocidade do fluido é maior na linha de centro e nula nas paredes, a interface água/óleo é curva e tende a se deslocar com maior facilidade pela região central do canal deixando um filme de óleo de $\sim 0,17 \mathrm{~mm}(\sim 0,17 \mathrm{~h})$ aderido nas paredes. Por conta disso a distância entre a parte mais avançada da interface e aquela representada pela linha de contato com a parede tende a aumentar com o tempo (Figura $5 a, b, c)$.

A presença da microcavidade cria uma distorção das linhas de corrente uma vez que na expansão abrupta, sem a parede superior que limite o movimento vertical do fluido, este tende a se expandir num típico fenômeno de die swell (Batchelor, Berry \& Horsfall, 1973; Tanner, 2005; Russo \& Phillips, 2010) originado pela diferença de tensões normais. Embora pouco acentuada, em todos os quadros da Figura 5 observa-se uma recirculação no sentido anti-horário na cavidade devido à transferência de momentum do fluido que escoa para o que fica retido. A recirculação quase simétrica enquanto a interface se encontra à montante da cavidade, ganha assimetria na medida que a interface atinge a cavidade e se desloca ao longo do canal de saída. 
Fração volumétrica de óleo

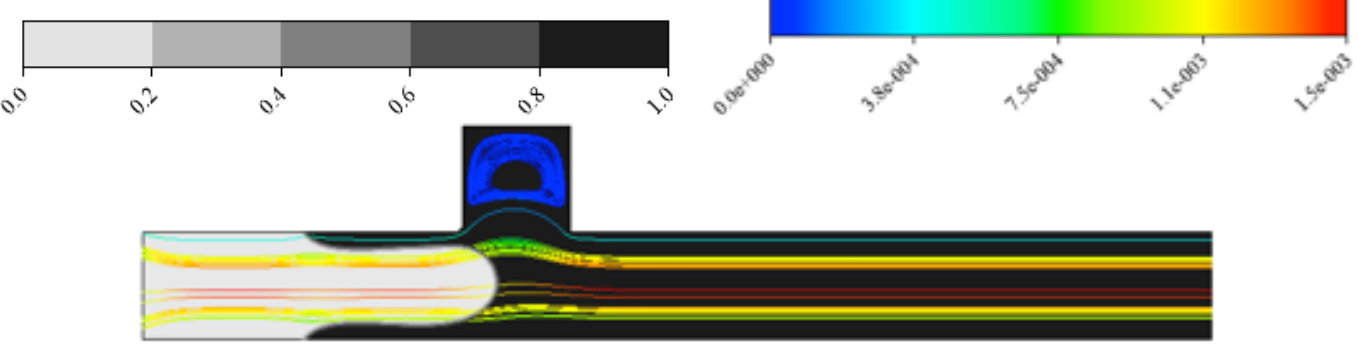

a) $t_{D}=0,276$

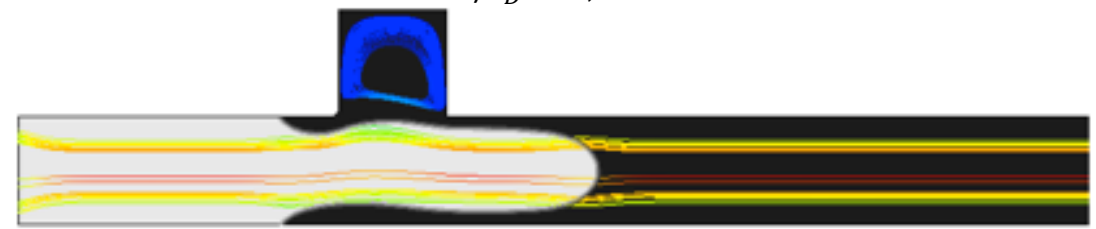

b) $t_{D}=0,460$

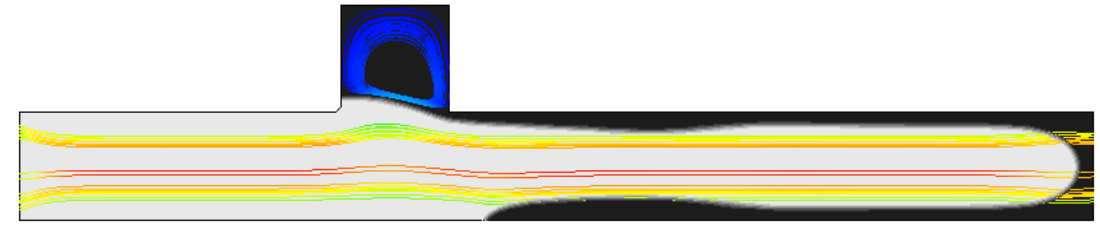

c) $t_{D}=0,828$

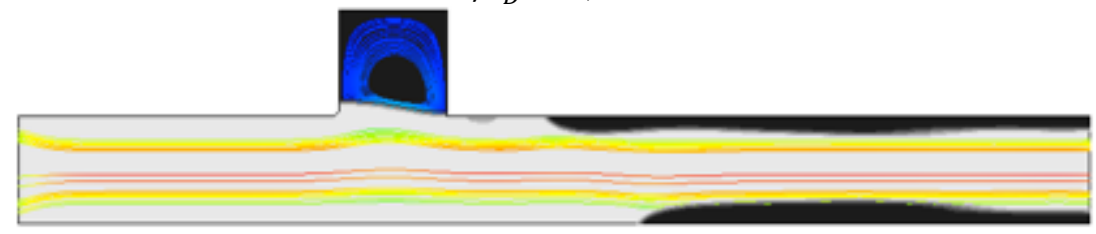

d) $t_{D}=1,104$

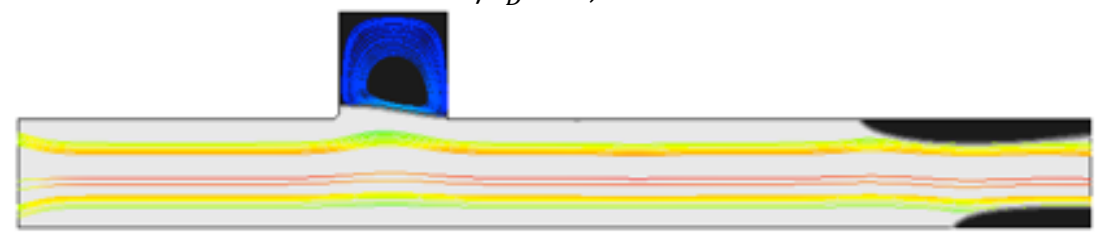

e) $t_{D}=1,656$

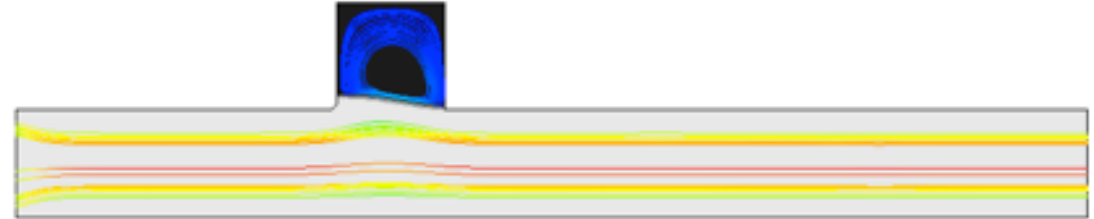

f) $t_{D}=2,300$

Figura 1: Fração volumétrica média de óleo para o caso 4 com raio da quina $r_{D}=10 \%$ e para tempos adimensionais representativos do processo de remoção de óleo.

Ao longo da curvatura da cavidade, a linha de contato tem movimento contínuo até se estabilizar pouco depois da região arredondada e no trecho inicial vertical da parede da cavidade, isso ocorre em $t_{D}=0,828$ (Figura $5 c$ ). A linha de contato fica, aparentemente, sem movimento devido a que nessa região a recirculação do fluido retido e o movimento do fluido injetado estão na mesma direção. Isso, por sua vez, cria uma região onde o escoamento é predominantemente extensional. Em diante a interface continua seu movimento até atingir o breakthrough (Figura $5 e$ ), e o processo passa a ser apenas para remover o óleo remanescente nas paredes superior e inferior do canal de saída. Na Figura 5e praticamente todo o óleo móvel foi retirado, a simulação 
entretanto é sustentada até $t_{D}=2,3$ (Figura $5 f$ ) para garantir que o volume retido represente efetivamente a saturação de óleo residual.

A primeira conclusão é que grande parte do óleo da cavidade fica trapeado. O óleo que foi retirado é quase inteiramente daquele que preenchia o canal. A sequência de imagens ilustra o impacto negativo da presença dos poros na eficiência do processo extrativo. Embora apenas um poro tenha sido considerado, a análise pode ser estendida para mais de um, em que o fenômeno virá, com suas particularidades, a ser repetido. A utilização de diferentes valores dos parâmetros adimensionais, como o número de capilaridade, causará alterações nestas respostas (Coelho et al., 2016).

O detalhe do escoamento na região da cavidade é mostrada na Figura 6 ao final da simulação, em $t_{D}=2,30$, e para quatro raios de curvatura da quina representada pelos casos 1 $\left(r_{D}=0 \%\right), 2\left(r_{D}=5 \%\right), 3\left(r_{D}=10 \%\right)$ e $4\left(r_{D}=25 \%\right)$, respectivamente. 0 raio adimensional $r_{D}$ é definido em função do raio da quina $r$ e do lado da cavidade $h$, como $r_{D}=r / h$.

Nos quatro casos uma grande quantidade de óleo permanece na cavidade. Entretanto, com o aumento do raio da quina a linha de contato desliza com mais facilidade ao longo da superfície sólida, e com isso a interface invade a cavidade de forma mais eficiente. Quinas agudas tendem a pinar a interface, dificultando sua movimentação. Como os resultados da figura são para o mesmo instante de tempo, conclui-se que para a maior raio de curvatura, a velocidade da linha de contato é maior. A Penetração na cavidade para $r_{D}=25 \%$ (Figura $6 \mathrm{~d}$ ) é praticamente o dobro do que o caso $r_{D}=0 \%$ (Figura 6a).

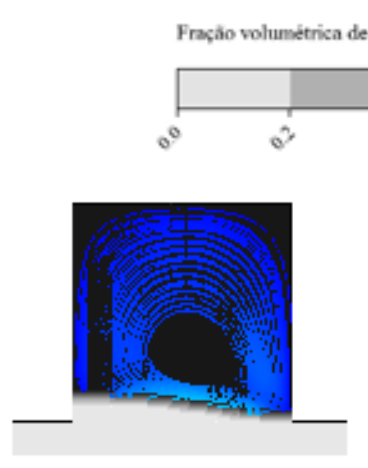

(a)

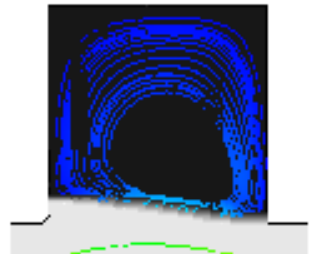

(b)

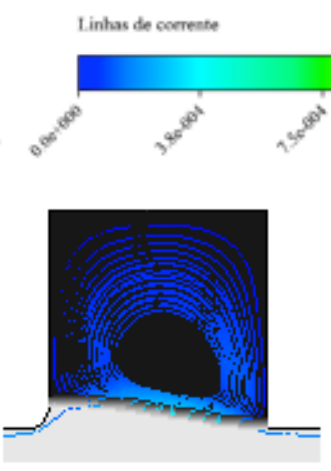

(c)

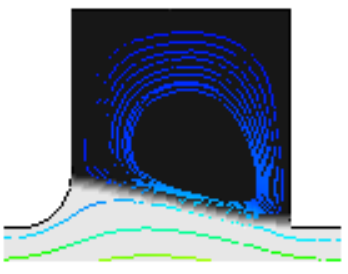

(d)

Figura 6: Influência da curvatura na fração volumétrica de óleo residual no tempo $t_{D}=2,30$ para (a) $r_{D}=0 \%$, (b) $r_{D}=5 \%$, (c) $r_{D}=10 \%$, (d) $r_{D}=25 \%$.

A resposta qualitativa mostrada e comentada nas Figuras 5 e 6 é apresentada de forma quantitativa nas Figura 7 e 8 utilizando a fração volumétrica média de óleo $\left(\bar{\alpha}_{o}\right)$ para diversos tempos adimensionais, $t_{D}$, e para os quatro raios de curvatura da quina já mencionados. 


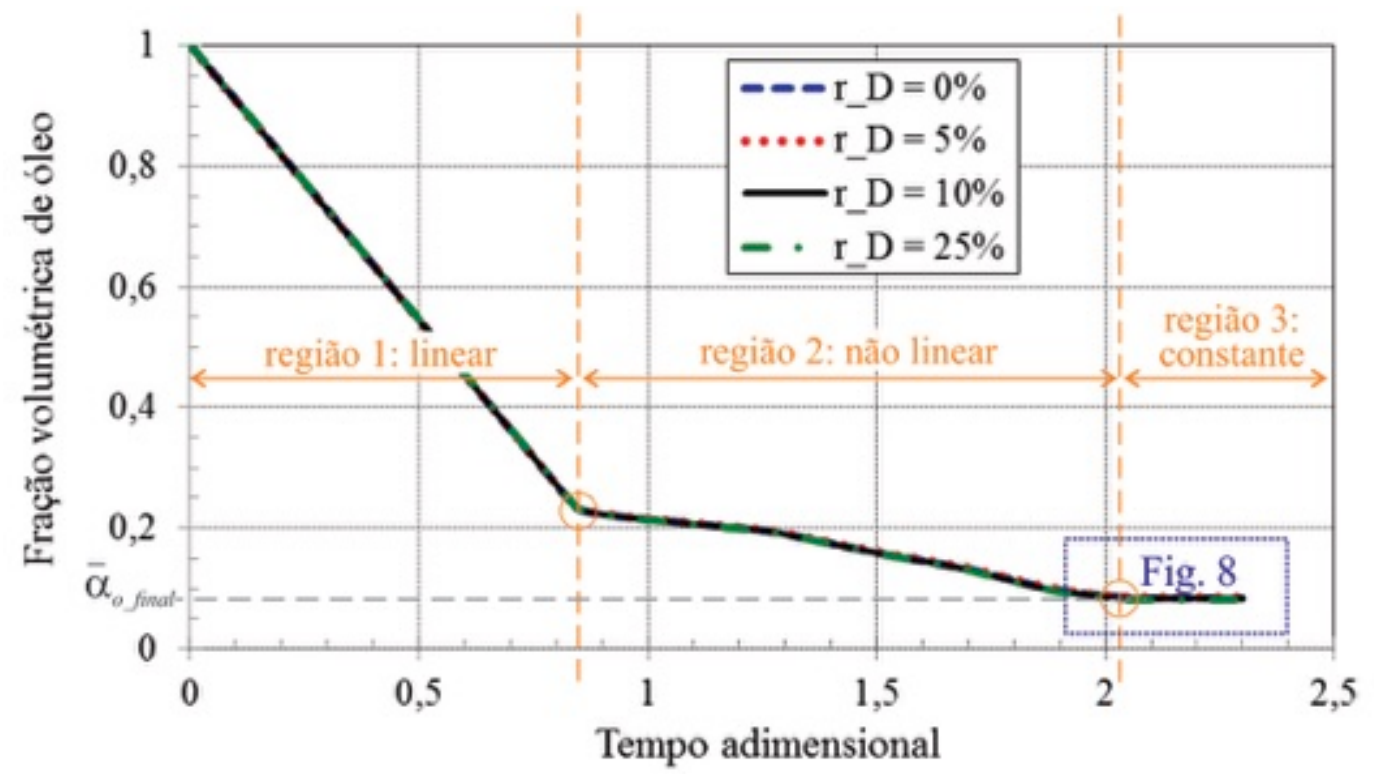

Figura 7. Relação da mobilização de óleo com o tempo adimensional para diferentes raios de curvatura.

A quantidade de óleo diminui com o tempo de injeção de água, sendo que este processo apresenta três regiões bem definidas. Uma região linear na qual a retirada de óleo é proporcional ao tempo de injeção. Uma segunda região não linear na qual a proporcionalidade não mais ocorre e cada vez menos óleo é retirado. A transição entre estas duas regiões ocorre em um tempo crítico $\mathrm{t}_{D}^{c 1}=0,844$, pouco depois da configuração da Figura 5 e para $t_{D}=0,828$. Esta mudança de regime acontece quando a interface água/óleo atinge o plano de saída, que na prática representa o breakthrough - BT. Este comportamento é explicado porque antes do BT apenas óleo é produzido, e a partir do BT água passa também a ser produzida junto com o óleo, com isso a quantidade de óleo movimentada diminui gradativamente e o da água aumenta. Esta afirmação é sustentada pela gráfico da Figura $5 \mathrm{~d}$ em $t_{D}=1,104$, na qual a parte frontal e mais avançada da interface está fora do domínio e o tempo corresponde à região não linear da figura. A terceira região é um platô praticamente constante que identifica a fração de óleo retida $\bar{\alpha}_{o_{-} \text {final }}$ no meio poroso e que não pode mais ser movimentado. É o limite inferior do intervalo da Equação (4), e que se mantem constante não mais sendo alterado pela continua injeção da água. A região 3 se inicia no tempo crítico $t_{D}^{c 2}$ e se estende até o final da simulação. Nesta escala todas as curvas aparentam ser coincidentes, entretanto o detalhe mostrado na Figura 8 destaca que não é assim.

As quatro curvas para os raios diferentes, apresentadas na Figura 7, não permitem visualizar, nesta escala, maiores alterações na resposta da fração volumétrica média de óleo. Entretanto de acordo com a Figura 8, que apresenta a fração de volume de óleo na região 3, $\bar{\alpha}_{o_{-} \text {final }}$, ao final da simulação $\left(t_{D}=2,30\right)$ para as quatro configurações consideradas, o aumento do raio de curvatura tem sim influência nos resultados. $O$ aumento do raio de curvatura da quina propicia uma maior retirada de óleo uma vez que a invasão da cavidade pela água é favorecida. 


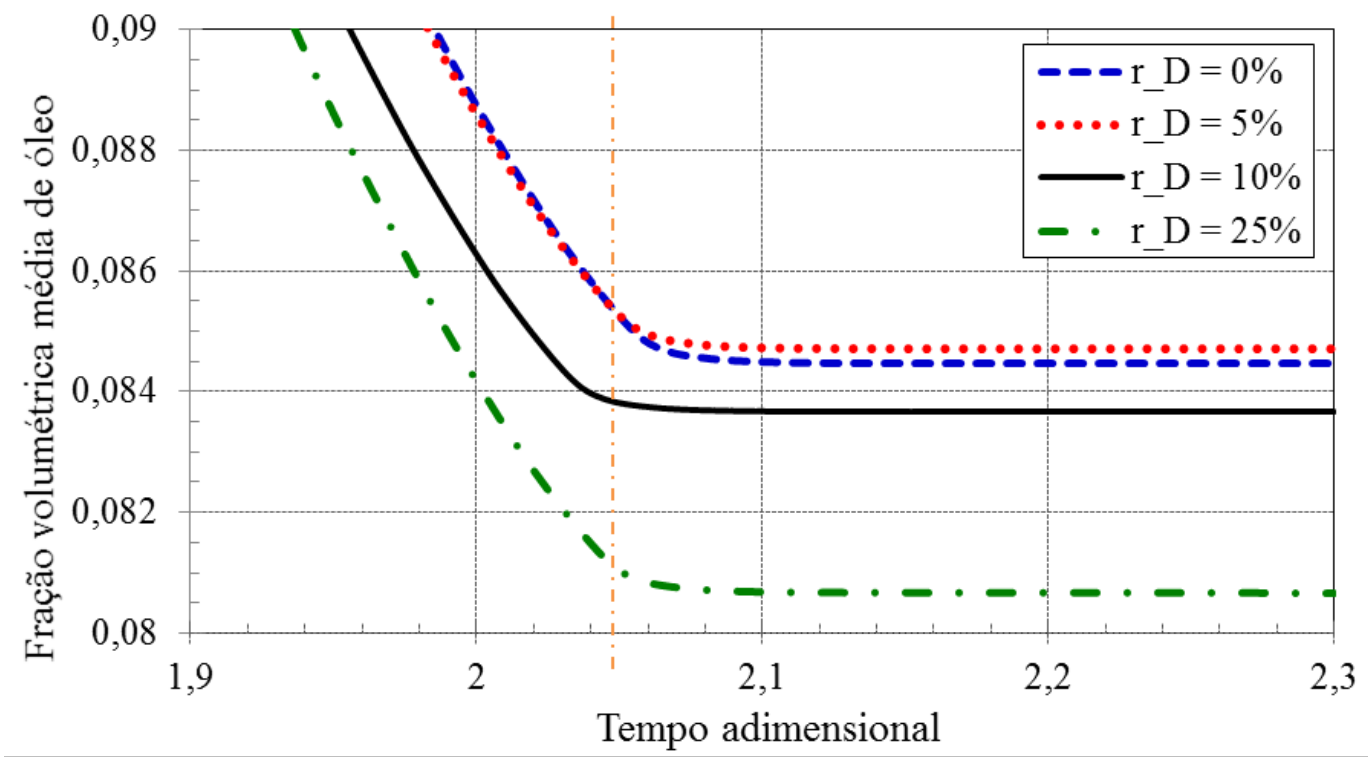

Figura 8. Relação da mobilização de óleo na microcavidade para os casos 1 até 4.

A partir de $t_{D}=2,1$, aproximadamente, a fração volumétrica média final de óleo assume um valor constante, independendo da continuidade da injeção de água. Como visto, a simulação continua até $t_{D}=2,3$.

Para os valores de raio $r$ adotados não existe grandes diferenças na quantidade de óleo que fica retido da microcavidade, analisando na região 3 . Isso pode ser causado porque as viscosidades do fluido deslocante (água) e do fluido deslocado (óleo) são iguais. As diferenças entre os casos 3 e 4 em relação ao caso base 1 são: $\bar{\alpha}_{o_{\text {final }_{3} 3}}-\bar{\alpha}_{o_{\text {final }_{1}}}=-8,10 \times 10^{-4}$, e $\bar{\alpha}_{o_{\text {final } 4}}-\bar{\alpha}_{o_{\text {final }_{1}}}=-31,28 \times 10^{-4}$. A redução da fração de óleo com o aumento do raio de curvatura é praticamente linear. A resposta para o raio adimensional de $5 \%$ (caso 2), comparado com a quina aguda, é de $\bar{\alpha}_{\text {final } 2_{2}}-\bar{\alpha}_{o_{\text {final }}}=+2,22 \times 10^{-4}$, oposto ao observado nos casos 3 e 4. A explicação é por que sendo valores muito pequenos e com variações na quinta casa decimal, a resposta se encontra dominada pelos erros oriundos do processo computacional.

\section{CONCLUSÕES}

Os resultados obtidos para o caso com quina aguda são coincidentes com os apresentados por Coelho et al. (2016). A análise dos quatro casos considerados permitem destacar as seguintes resultados:

a) Ao final do processo de deslocamento uma quantidade de óleo continuará trapeado na microcavidade;

b) Para a geometria considerada e independente do raio de curvatura da quina, existem três regiões distintas ao se avaliar a fração volumétrica média de óleo. A primeira região, antes do $\mathrm{BT}$, em que a redução de óleo é acentuada. Uma segunda região onde a retirada de óleo torna-se cada vez mais lenta. E uma terceira que identifica a saturação de óleo residual;

c) O processo se torna mais eficiente quanto maior for o raio de curvatura da quina do microporo. Esta relação, pelo menos para os casos analisados, é linear. 


\section{REFERÊNCIAS}

Batchelor J., Berry J.P., \& Horsfall F. (1973). Die swell in elastic and viscous fluids. Polymer, v.14, p.297-299,

Bohacek, J. (2010). Surface tension model for high viscosity ratios implemented in VOF model, 23rd annual conference on liquid atomization and spray systems, p. 1-8.

Brackbill, J.U., Kothe, D.B., \& Zemach, C. (1992). A continuum method for modeling surface tension. Journal of Computational Physics, v.100, n.2, p.335-354. DOI: dx.doi.org/10.1016/0021-9991(92)90240-y.

Coelho, J.K., Pena, M.D., \& Romero, O.J. (2016). Pore-scale modeling of oil mobilization trapped in a square cavity. IEEE America Latina, v.14, n.4, p.1800-1807. DOI: 10.1109/TLA.2016.7483518.

Galusinski, C., \& Vigneaux, P. (2008). On stability condition for bifluid flows with surface tension: application to microfluidics. Journal of Computational Physics, v. 227, p. 6140-6164. DOI: 10.1016/j.jcp.2008.02.023.

Green D.W., \& Willhite G.P. (1998). Enhanced oil recovery. Richardson, Texas: henry I. Doherty memorial found for AIME society of petroleum engineers, $553 \mathrm{p}$.

Hirt, C.W., \& Nichols, B.D. (1981). Volume of fluid (VOF) method for the dynamics of free boundaries. Journal of Computational Physics, v.39, n.1, p.201-225. DOI: dx.doi.org/10.1016/0021-9991(81)90145-5.

Kamyabi, A., Ramazani, A.S.A., \& Kamyabi, M.M. (2015). Surfactant effects on the efficiency of oil sweeping from the dead ends: numerical simulation and experimental investigation. Chemical Engineering Research and Design, v. 94, p. 173-181. DOI: 10.1016/j.cherd.2014.07.027.

Kamyabi, A., Ramazani, A.S.A., \& Kamyabib, M.M. (2013). Effects of viscoelastic polymer solutions on the oil extraction from dead ends. Scientia Iranica C, v.20, p.1912-1920.

McCain Jr., W.D. (1990). The properties of petroleum fluids, second edition. PennWell Books, Tulsa, Oklahoma.

Pena, M.D., Romero, O.J., \& Ribeiro, D.C. (2014). Mobilização de óleo retido em microcavidades mediante injeção de fluidos não-newtonianos inelásticos. Latin American Journal Of Energy Research, v.1, n. 1, p. 30-38, jun. DOI: 10.21712/lajer.2014.v1.n1.p30-38.

Romero O.J., \& Fejoli R.F. (2015). Fluxo de uma gota de óleo em um canal reto e com garganta. Revista Iberoamericana de Ingeniería Mecánica, v.19, p.71-81.

Romero O.J., Scriven L.E., \& Carvalho M.S. (2006). Effect of curvature of coating die edges on the pinning of contact line. AIChe Journal, v.52, p.447-455. DOI:10.1002/aic.10672.

Russo G., \& Phillips, T.N. (2010). Numerical simulation of steady planar die swell for a Newtonian fluid using the spectral element method. Computers \& fluids, v.39, p.780-792. 
Santos, K.B., Romero, O.J., Meneguelo, A.P., \& Ribeiro, D.C. (2016). A numerical investigation of immiscible water-oil displacement in simplified porous media. IEEE America Latina, v.14, n.5, p.2175 - 2183. DOI: 10.1109/TLA.2016.7530411.

Tanner, R.I. (2005). A theory of die-swell revisited. J. Non-Newtonian Fluid Mechanics, v.129, p.85-87.

Yin, H., Wang, D., \& Zhong, H. (2006). Study flow behaviors of viscoelastic polymer solution in micropore with dead end. Paper SPE 101950 presented at 2006 SPE annual technical conference and exhibition. San Antonio, Texas, USA. DOI: 10.2118/101950-ms. 